\title{
NON-ABELIAN TENSOR AND EXTERIOR PRODUCTS MODULO $q$ AND UNIVERSAL $q$-CENTRAL RELATIVE EXTENSION OF LIE ALGEBRAS
}

\author{
EMZAR KHMALADZE
}

(communicated by Graham Ellis)

\begin{abstract}
The notions of tensor end exterior products modulo $q$ of two crossed $P$-modules, where $q$ is a positive integer and $P$ is a Lie algebra, are introduced and some properties are established. The condition for the existence of a universal $q$-central relative extension of a Lie epimorphism is given and this extension is described as an exterior product modulo $q$.
\end{abstract}

\section{Introduction}

The non-abelian tensor product of groups was introduced by Brown and Loday [3,4] and has applications in homotopy theory and in non-abelian (co)homology theory of groups $[11,13,14]$.

In [5] Conduche and Rodriguez-Fernandez introduce the non-abelian tensor product modulo an integer $q$ of groups, generalizing definitions of Brown [2] and Ellis and Rodriguez [9]. This construction is the mod $q$ version of the non-abelian tensor product of groups of Brown and Loday.

In [6] Ellis developed an analogous theory of non-abelian tensor product for Lie algebras (see also [7]). Using tensor (exterior) product of Lie algebras Ellis describes the universal central extension of Lie algebras. The importance of this product is given by Guin in [12], constructing the non-abelian homology of Lie algebras in low dimensions, which has applications in cyclic homology.

In the present paper we introduce the non-abelian tensor (exterior) product modulo $q$, $M \otimes^{q} N\left(M \wedge^{q} N\right)$, where $M$ and $N$ are two crossed $P$-modules, in the context of Lie algebras, as the $\bmod q$ version of Ellis' tensor (exterior) product of Lie algebras and investigate its properties. The general aim introducing this notion is to describe the universal $q$-central relative extension of a Lie epimorphism, analogously to Conduche-Rodriguez-Fernandez's result in the group case [5, Theorem 2.11].

In [16] Kassel and Loday give the notion of relative extension of a Lie epimorphism $\alpha: P \rightarrow Q$ and prove that a universal central relative extension exists if and only if the relative homology group $H_{2}(Q, P ; \Lambda)=0$, where $\Lambda$ is a principal ring. In this paper we introduce the definition of a $q$-central relative extension of a Lie epimorphism, which is the mod $q$ version of Kassel-Loday's notion and give our main result (Theorem 2.8): for a short exact sequence of Lie algebras

$$
0 \rightarrow N \rightarrow P \stackrel{\alpha}{\rightarrow} Q \rightarrow 0
$$

there exists a universal $q$-central relative extension of $\alpha$ if and only if $N=N \#_{q} P$, where $N \#_{q} P$ is the submodule of $P$ generated by the elements $[n, p]$ and $q n$ for $n \in N, p \in P$.

The work was partially supported by INTAS Georgia grant No 213 and NATO linkage grant No 975316 . The author also would like to thank N. Inassaridze and the referee for helpful comments

Received 5 July 1999, revised 15 November 1999; published on 29 November 1999.

1991 Mathematics Subject Classification: 18G50

Key words and phrases: Lie algebra, tensor product, exterior product, crossed module, universal extension

(C) 1999, Emzar Khmaladze. Permission to copy for private use granted. 
In the rest of the paper the following interesting properties of non-abelian tensor (exterior) product modulo $q, M \otimes^{q} N\left(M \wedge^{q} N\right)$, of Lie algebras are given. The existence of a unique isomorphism $M \otimes^{q} N \rightarrow N \otimes^{q} M\left(M \wedge^{q} N \rightarrow N \wedge^{q} M\right)$ (Proposition 1.7) is shown. Compatibility of tensor product modulo $q$ of crossed modules with the direct limit of crossed modules is established (Theorem 1.9). Some examples of crossed squares of Lie algebras are given. Using a slightly generalized version of Whitehead's universal quadratic functor (for the definition see bellow) the relation between the Lie exterior product modulo $q$ and the Lie tensor product modulo $q$ is estabished (Theorem 1.17). Finally, the relation between Ellis' non-abelian tensor product and the non-abelian tensor product modulo $q$ of two Lie algebras with compatible actions on each other is given (Theorem 1.22).

Notation. We shall use the term Lie algebra to mean a Lie algebra over $\Lambda$, where $\Lambda$ is a commutative ring with identity. We denote by [,] the Lie bracket and by $q$ a non-negative integer. For any Lie algebra $X$, an ideal $Y \subseteq X$ and $x \in X$ we shall write $c l(x)$ to denote the coset $x+Y$.

\section{Tensor and exterior products modulo $q$ of Lie algebras}

Let $P$ and $M$ be two Lie algebras. By an action of $P$ on $M$ we mean a $\Lambda$-bilinear map $P \times M \rightarrow M,(p, m) \mapsto{ }^{p} m$ satisfying

$$
\begin{aligned}
{ }^{\left[p, p^{\prime}\right]} m & ={ }^{p}\left(p^{p^{\prime}} m\right)-{ }^{p^{\prime}}\left({ }^{p} m\right), \\
{ }^{p}\left[m, m^{\prime}\right] & =\left[{ }^{p} m, m^{\prime}\right]+\left[m,{ }^{p} m^{\prime}\right]
\end{aligned}
$$

for all $m, m^{\prime} \in M, p, p^{\prime} \in P$. Note that any Lie algebra acts on its ideals by Lie multiplication.

Recall from [16] (see also [6]) that, in the context of Lie algebras, a crossed $P$-module is a Lie homomorphism $\mu: M \rightarrow P$ together with an action of $P$ on $M$ which satisfies the following conditions:

(i) $\mu\left({ }^{p} m\right)=[p, \mu(m)]$,

(ii) ${ }^{\mu(m)} m^{\prime}=\left[m, m^{\prime}\right]$

for all $m, m^{\prime} \in M, p \in P$.

A morphism of crossed modules $\mu: M \rightarrow P$ and $\mu^{\prime}: M^{\prime} \rightarrow P^{\prime}$ is a pair $\left(f: M \rightarrow M^{\prime}\right.$, $\left.\varphi: P \rightarrow P^{\prime}\right)$ of Lie homomorphisms such that $f\left({ }^{p} m\right)=\varphi(p) f(m)$ for all $m \in M, p \in P$ and $\mu^{\prime} f=\varphi \mu$.

Suppose that $\mu: M \rightarrow P$ and $\nu: N \rightarrow P$ are two crossed $P$-modules and consider the pullback

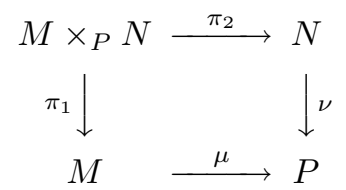

Let $K=M \times{ }_{P} N=\{(m, n) \in M \times N \mid m \in M, n \in N, \mu(m)=\nu(n)\}$. In this diagram each Lie algebra acts on any other via its image in the Lie algebra $P$.

Definition 1.1. The tensor product modulo $q, M \otimes^{q} N$, of the crossed $P$-modules $\mu$ and $\nu$ is the Lie algebra generated by the symbols $m \otimes n$ and $\{k\}, m \in M, n \in N, k \in K$ subject to the following relations:

$$
\begin{gathered}
\lambda(m \otimes n)=\lambda m \otimes n=m \otimes \lambda n \\
\left(m+m^{\prime}\right) \otimes n=m \otimes n+m^{\prime} \otimes n, \\
m \otimes\left(n+n^{\prime}\right)=m \otimes n+m \otimes n^{\prime},
\end{gathered}
$$




$$
\begin{array}{r}
{\left[m, m^{\prime}\right] \otimes n=m \otimes{ }^{m^{\prime}} n-m^{\prime} \otimes{ }^{m} n,} \\
m \otimes\left[n, n^{\prime}\right]={ }^{{ }^{\prime}} m \otimes n-{ }^{n} m \otimes n^{\prime}, \\
{\left[m \otimes n, m^{\prime} \otimes n^{\prime}\right]=-{ }^{n} m \otimes{ }^{m^{\prime}} n^{\prime},} \\
{[\{k\}, m \otimes n]={ }^{q k} m \otimes n+m \otimes{ }^{q k} n,} \\
\left\{\lambda k+\lambda^{\prime} k^{\prime}\right\}=\lambda\{k\}+\lambda^{\prime}\left\{k^{\prime}\right\}, \\
{\left[\{k\},\left\{k^{\prime}\right\}\right]=\pi_{1}(q k) \otimes \pi_{2}\left(q k^{\prime}\right),} \\
\left\{\left(-{ }^{n} m,{ }^{m} n\right)\right\}=q(m \otimes n)
\end{array}
$$

for all $m, m^{\prime} \in M, n, n^{\prime} \in N, k, k^{\prime} \in K, \lambda, \lambda^{\prime} \in \Lambda$.

Let $M \square N$ be the submodule of $M \otimes^{q} N$ generated by the elements $m \otimes n$ with $\mu(m)=\nu(n)$. Then $M \square N$ lies in the centre of $M \otimes^{q} N$ since for any $m \otimes n \in M \square N, m^{\prime} \otimes n^{\prime} \in M \otimes N$ we have $\left[m \otimes n, m^{\prime} \otimes n^{\prime}\right]=0$ (see [6]) and for any $\{k\} \in K$ by relations (1.3), (1.5) one has

$$
\begin{aligned}
& {[\{k\}, m \otimes n]={ }^{q k} m \otimes n+m \otimes{ }^{q k} n=\left[\pi_{1}(q k), m\right] \otimes n+m \otimes{ }^{q k} n} \\
& =\pi_{1}(q k) \otimes{ }^{m} n-m \otimes{ }^{q k} n+m \otimes{ }^{q k} n=\pi_{1}(q k) \otimes{ }^{\mu(m)} n \\
& =\pi_{1}(q k) \otimes{ }^{\nu(n)} n=\pi_{1}(q k) \otimes[n, n]=0 .
\end{aligned}
$$

In particular, $M \square N$ is an ideal of $M \otimes^{q} N$.

Definition 1.2. The exterior product modulo $q, M \wedge^{q} N$, of the crossed P-modules $\mu$ and $\nu$ is the quotient

$$
M \otimes \otimes^{q} N / M \square N .
$$

In other words, the Lie algebra $M \wedge^{q} N$ is the quotient of the Lie algebra $M \otimes^{q} N$ by the relation

$$
\pi_{1}(k) \otimes \pi_{2}(k)=0, \quad k \in K .
$$

Let us denote by $m \wedge n$ the image of $m \otimes n$ in $M \wedge^{q} N$.

Proposition 1.3. There are two Lie homomorphisms $\xi: M \otimes^{q} N \rightarrow M$ and $\xi^{\prime}: M \otimes^{q} N \rightarrow N$ defined by

$$
\begin{aligned}
& \xi(m \otimes n)=-{ }^{n} m, \quad \xi(\{k\})=\pi_{1}(q k), \\
& \xi^{\prime}(m \otimes n)={ }^{m} n, \quad \xi^{\prime}(\{k\})=\pi_{2}(q k) .
\end{aligned}
$$

Moreover, these homomorphisms factor through $M \wedge^{q} N$.

Proof. [6, Proposition 2] leaves us to show that $\xi$ and $\xi^{\prime}$ commute with relations (1.5)-(1.9). In effect,

$$
\begin{aligned}
& \xi\left({ }^{q k} m \otimes n+m \otimes{ }^{q k} n\right)=-{ }^{n}\left({ }^{q k} m\right)-{ }^{\left({ }^{q k} n\right)} m=-{ }^{n}\left({ }^{q k} m\right)-{ }^{\nu\left({ }^{\mu \pi_{1}(q k)} n\right)} m \\
& =-{ }^{n}\left({ }^{q k} m\right)-\left[{ }^{\left.\mu \pi_{1}(q k), \nu(n)\right]} m=-{ }^{n}\left({ }^{q k} m\right)-{ }^{\mu \pi_{1}(q k)}\left({ }^{n} m\right)+{ }^{n}\left({ }^{q k} m\right)\right. \\
& =-\left[\pi_{1}(q k),{ }^{n} m\right]=\xi([\{k\}, m \otimes n]) .
\end{aligned}
$$

The proof of the rest is left as an exercise.

Remark 1.4. There is the canonical Lie homomorphism $\delta: M \otimes^{q} N \rightarrow M \times_{P} N$ (resp. $\left.\delta^{\prime}: M \wedge^{q} N \rightarrow M \times_{P} N\right)$, given, for $x \in M \otimes^{q} N$ (resp. $\left.x \in M \wedge^{q} N\right)$ by $\delta(x)=\left(\xi(x), \xi^{\prime}(x)\right)$ (resp. $\delta^{\prime}(x)=\left(\xi(x), \xi^{\prime}(x)\right)$ ). In the case $q=1$ the map $\delta$ is an isomorphism of Lie algebras.

Lemma 1.5. (i) Let $m, m^{\prime}, m^{\prime \prime} \in M$ and $n, n^{\prime}, n^{\prime \prime} \in N$ be such that $\mu(m)=\nu(n)=\nu\left(n^{\prime \prime}\right)$ and $\mu\left(m^{\prime}\right)=\mu\left(m^{\prime \prime}\right)=\nu\left(n^{\prime}\right)$, then

$$
q m^{\prime \prime} \otimes q n^{\prime \prime}=-q m \otimes q n^{\prime}=q m^{\prime} \otimes q n .
$$


(ii) Let $k, k^{\prime} \in K$ and suppose $\left[k, k^{\prime}\right]=0$, then

$$
q\left(\pi_{1}(k) \otimes \pi_{2}\left(k^{\prime}\right)\right)=0
$$

Proof. (i) By the relation (1.7) one has

$$
\begin{aligned}
& q m^{\prime \prime} \otimes q n^{\prime \prime}=\left[\left\{\left(m^{\prime \prime}, n^{\prime}\right)\right\},\left\{\left(m, n^{\prime \prime}\right)\right\}\right]=-\left[\left\{\left(m, n^{\prime \prime}\right)\right\},\left\{\left(m^{\prime \prime}, n^{\prime}\right)\right\}\right] \\
& =-q m \otimes q n^{\prime}=-\left[\{(m, n)\},\left\{\left(m^{\prime}, n^{\prime}\right)\right\}\right]=q m^{\prime} \otimes q n .
\end{aligned}
$$

(ii) Follows from the relation (1.8) and the fact that $\{0\}=0$.

Recall from [6] Ellis' original definition of the non-abelian tensor product of Lie algebras, $M \otimes N$, which is the Lie algebra generated by elements $m \otimes n, m \in M, n \in N$ and subject to the relations (1.1)-(1.4). Furthermore, Ellis' exterior product, $M \wedge N$, is the Lie algebra generated by elements $m \wedge n, m \in M, n \in N$ and subject to the relations (1.1)-(1.4) and (1.9) (see $[6],[7])$

Let $[M, N]$ be the submodule of $K=M \times{ }_{P} N$ generated by the elements $\left(-{ }^{n} m,{ }^{m} n\right)$, $m \in M, n \in N$. It is easy to see that $[M, N]$ is an ideal of $K$. Further, $[M, N]$ contains the commutator $[K, K]$ of $K$ since for $k, k^{\prime} \in K$ one has

$$
\left[k, k^{\prime}\right]=\left(-{ }^{\pi_{2}\left(k^{\prime}\right)} \pi_{1}(k),{ }^{\pi_{1}(k)} \pi_{2}\left(k^{\prime}\right)\right) .
$$

We have the following

Proposition 1.6. There is a commutative diagram of Lie algebras

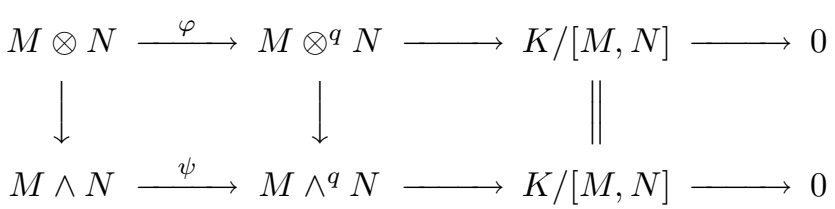

with exact rows.

Proof. At first note that the Lie algebra $K /[M, N]$ is abelian. The homomorphism $\varphi$ (resp. $\psi)$ is defined by $\varphi(m \otimes n)=m \otimes n($ resp. $\psi(m \wedge n)=m \wedge n)$. By (1.5) $\operatorname{Im} \varphi($ resp. $\operatorname{Im} \psi)$ is an ideal of $M \otimes^{q} N$ (resp. $\left.M \wedge^{q} N\right)$. It is clear that the quotient of $M \otimes^{q} N\left(\operatorname{resp} . M \wedge^{q} N\right)$ by $\varphi(M \otimes N)$ (resp. $\psi(M \wedge N)$ ) is generated by elements $\{k\}, k \in K$ with the relations $\left\{\lambda k+\lambda^{\prime} k^{\prime}\right\}=\lambda\{k\}+\lambda^{\prime}\left\{k^{\prime}\right\},\left[\{k\},\left\{k^{\prime}\right\}\right]=0,\left\{\left(-{ }^{n} m,{ }^{m} n\right)\right\}=0$ and the diagram is commutative.

The tensor and exterior products of Lie algebras modulo $q$ are symmetric as we shall show now.

Proposition 1.7. Let $(M, \mu)$ and $(N, \nu)$ be crossed P-modules. Then there is a unique isomorphism of Lie algebras

$$
s: M \otimes^{q} N \longrightarrow N \otimes^{q} M \quad\left(s: M \wedge^{q} N \longrightarrow N \wedge^{q} M\right),
$$

such that $s(m \otimes n)=-(n \otimes m)(s(m \wedge n)=-(n \wedge m)), s(\{k\})=\{\bar{k}\}$, where $\bar{k}=\left(\pi_{2}(k), \pi_{1}(k)\right)$ for all $m \in M, n \in N$ and $k \in K$.

Proof. We have only to show that $s$ commutes with relations (1.5)-(1.9) (for relations (1.1)(1.4) see [6]). In effect,

$$
\begin{gathered}
s([\{k\}, m \otimes n])=[\{\bar{k}\},-n \otimes m]=-\left({ }^{q \bar{k}} n \otimes m+n \otimes{ }^{q \bar{k}} m\right) \\
=-\left({ }^{q k} n \otimes m+n \otimes{ }^{q k} m\right)=s\left({ }^{q k} m \otimes n+m \otimes{ }^{q k} n\right), \\
s\left(\left\{\lambda k+\lambda^{\prime} k^{\prime}\right\}\right)=\left\{\overline{\lambda k+\lambda^{\prime} k^{\prime}}\right\}=\lambda\{\bar{k}\}+\lambda^{\prime}\left\{\overline{k^{\prime}}\right\}=s\left(\lambda\{k\}+\lambda^{\prime}\left\{k^{\prime}\right\}\right) .
\end{gathered}
$$


By Lemma 1.5(i) one has

$$
\begin{gathered}
s\left(\left[\{k\},\left\{k^{\prime}\right\}\right]\right)=\left[\{\bar{k}\},\left\{\overline{k^{\prime}}\right\}\right]=\pi_{1}(q \bar{k}) \otimes \pi_{2}\left(q \overline{k^{\prime}}\right) \\
=\pi_{2}\left(q k^{\prime}\right) \otimes \pi_{1}(q k)=s\left(\pi_{1}(q k) \otimes \pi_{2}(q k)\right), \\
s\left(\left\{\left(-{ }^{m} n,{ }^{m} n\right)\right\}\right)=\left\{\left({ }^{m} n,-{ }^{n} m\right)\right\}=-q(n \otimes m)=s(q(m \otimes n)) .
\end{gathered}
$$

And finally

$$
s\left(\pi_{1}(k) \wedge \pi_{2}(k)\right)=-\left(\pi_{2}(k) \wedge \pi_{1}(k)\right)=0 . \square
$$

Proposition 1.8. Let $(M, \mu),(N, \nu)$ be crossed P-modules and $\left(M^{\prime}, \mu^{\prime}\right),\left(N^{\prime}, \nu^{\prime}\right)$ be crossed $P^{\prime}$-modules. Suppose $\alpha=(f, \varphi):(M, \mu) \rightarrow\left(M^{\prime}, \mu^{\prime}\right), \beta=(g, \psi):(N, \nu) \rightarrow\left(N^{\prime}, \nu^{\prime}\right)$ are crossed module morphisms such that $\varphi=\psi$. Then there are natural homomorphisms of Lie algebras

$$
\begin{gathered}
\alpha \otimes^{q} \beta: M \otimes^{q} N \longrightarrow M^{\prime} \otimes^{q} N^{\prime} \\
\left(\alpha \wedge^{q} \beta: M \wedge^{q} N \longrightarrow M^{\prime} \wedge^{q} N^{\prime}\right),
\end{gathered}
$$

such that $(\alpha \otimes \beta)(m \otimes n)=f(m) \otimes g(n)\left(\left(\alpha \wedge^{q} \beta\right)(m \wedge n)=f(m) \wedge g(n)\right),\left(\alpha \otimes^{q} \beta\right)(\{k\})=$ $\left\{\left(f \pi_{1}(k), g \pi_{2}(k)\right)\right\}\left(\left(\alpha \wedge^{q} \beta\right)(\{k\})=\left\{\left(f \pi_{1}(k), g \pi_{2}(k)\right)\right\}\right)$ for all $m \in M, n \in N$ and $k \in K$. Furthermore, if $\alpha, \beta$ are onto, so also is $\alpha \otimes^{q} \beta\left(\alpha \wedge^{q} \beta\right)$.

Proof. Note that $\left(f \pi_{1}(k), g \pi_{2}(k)\right) \in M^{\prime} \times_{P^{\prime}} N^{\prime}$ for all $k \in K=M \times_{P} N . \alpha \otimes^{q} \beta$ plainly commutes with relations (1.1)-(1.9). For instance,

$$
\begin{aligned}
& \left(\alpha \otimes^{q} \beta\right)([\{k\}, m \otimes n])=\left[\left\{\left(f \pi_{1}(k), g \pi_{2}(k)\right)\right\}, f(m) \otimes g(n)\right] \\
& ={ }^{\mu^{\prime} f \pi_{1}(q k)} f(m) \otimes g(n)+f(m) \otimes{ }^{\nu^{\prime} g \pi_{2}(q k)} g(n) \\
& ={ }^{\varphi \mu \pi_{1}(q k)} f(m) \otimes g(n)+f(m) \otimes{ }^{\varphi \nu \pi_{2}(q k)} g(n) \\
& =f\left({ }^{\mu \pi_{1}(q k)} m\right) \otimes g(n)+f(m) \otimes g\left({ }^{\nu \pi_{2}(q k)} n\right) \\
& =\left(\alpha \otimes^{q} \beta\right)\left({ }^{q k} m \otimes n+m \otimes{ }^{q k} n\right) . \square
\end{aligned}
$$

Now we investigate the compatibility of the tensor product modulo $q, \otimes^{q}$, with the direct limit of crossed modules. The group-theoretic version of this result is given in [15].

Theorem 1.9. Let $\left\{M_{\alpha}, \Phi_{\alpha}^{\beta}, \alpha \leqslant \beta\right\}$ and $\left\{P_{\alpha}, \Psi_{\alpha}^{\beta}, \alpha \leqslant \beta\right\}$ be two directed systems of Lie algebras. Let $\mu_{\alpha}: M_{\alpha} \rightarrow P_{\alpha}$ be a crossed $P_{\alpha}$-module for each $\alpha$ such that $\left(\Phi_{\alpha}^{\beta}, \Psi_{\alpha}^{\beta}\right):\left(M_{\alpha}, \mu_{\alpha}\right) \rightarrow$ $\left(M_{\beta}, \nu_{\beta}\right), \alpha \leqslant \beta$ is a crossed module morphism. Let $\nu_{\alpha}: N \rightarrow P_{\alpha}$ be a crossed $P_{\alpha}$ - module for each $\alpha$ such that $\left(1, \Psi_{\alpha}^{\beta}\right):\left(N, \nu_{\alpha}\right) \rightarrow\left(N, \nu_{\beta}\right), \alpha \leqslant \beta$ is a crossed module morphism. Then there are natural isomorphisms of Lie algebras

$$
\left.\left(\varliminf_{\alpha}\left\{M_{\alpha}\right\}\right) \otimes^{q} N \approx \varliminf_{\lim _{\alpha}}\left\{M_{\alpha} \otimes^{q} N\right\}, \quad \varliminf_{\alpha}\left\{M_{\alpha}\right\}\right) \wedge^{q} N \approx \varliminf_{\alpha}\left\{M_{\alpha} \wedge^{q} N\right\},
$$

where $\varliminf_{\alpha}\left\{M_{\alpha}\right\} \otimes^{q} N$ is considered as the tensor product modulo $q$ of crossed $\varliminf_{\alpha}\left\{P_{\alpha}\right\}$ modules.

Proof. It is easy to check that a homomorphism $\nu: N \rightarrow \varliminf_{\alpha}\left\{P_{\alpha}\right\}$ defined by $\nu(n)=$ $\operatorname{cl}\left(\nu_{\alpha}(n)\right), n \in N$ with an action ${ }^{c l\left(p_{\alpha}\right)} n=p_{\alpha} n$ is a crossed module.

Let $\mu: \varliminf_{\alpha}\left\{M_{\alpha}\right\} \rightarrow \varliminf_{\alpha}\left\{P_{\alpha}\right\}$ be the Lie homomorphism defined by $\mu\left(\operatorname{cl}\left(m_{\alpha}\right)\right)=$ $c l\left(\mu_{\alpha}\left(m_{\alpha}\right)\right)$. There is an action of $\lim _{\alpha}\left\{P_{\alpha}\right\}$ on $\lim _{\alpha}\left\{M_{\alpha}\right\}$ defined by ${ }^{c l}\left(p_{\alpha}\right) c l\left(m_{\beta}\right)=$ $c l\left(\Psi_{\alpha}^{\gamma}\left(p_{\alpha}\right) \Phi_{\beta}^{\gamma}\left(m_{\beta}\right)\right)$, where $\gamma \geqslant \alpha, \beta$ (the existense of such $\gamma$ follows from the directeness of the system). It is easy to check that everything is well defined here. Then we have

$$
\begin{aligned}
& \mu\left({ }^{c l\left(p_{\alpha}\right)} \operatorname{cl}\left(m_{\beta}\right)\right)=\mu\left(\operatorname{cl}\left({ }^{\Psi_{\alpha}^{\gamma}\left(p_{\alpha}\right)} \Phi_{\beta}^{\gamma}\left(m_{\beta}\right)\right)\right)=\operatorname{cl}\left(\mu_{\gamma}\left({ }^{\Psi_{\alpha}^{\gamma}\left(p_{\alpha}\right)} \Phi_{\beta}^{\gamma}\left(m_{\beta}\right)\right)\right) \\
& =\operatorname{cl}\left(\left[\Psi_{\alpha}^{\gamma}\left(p_{\alpha}\right), \mu_{\gamma}\left(\Phi_{\beta}^{\gamma}\left(m_{\beta}\right)\right)\right]\right)=\operatorname{cl}\left(\left[\Psi_{\alpha}^{\gamma}\left(p_{\alpha}\right), \Psi_{\beta}^{\gamma} \mu_{\beta}\left(m_{\beta}\right)\right]\right) \\
& \left.=\left[c l\left(p_{\alpha}\right), \mu_{(} c l\left(m_{\beta}\right)\right)\right] \text {, where } \gamma \geqslant \alpha, \beta \text {; }
\end{aligned}
$$




$$
\begin{aligned}
& \mu\left(c l\left(m_{\alpha}\right)\right) \\
& =c l\left(m_{\beta}\right)={ }^{c l\left(\mu_{\alpha}\left(m_{\alpha}\right)\right)} \operatorname{cl}\left(m_{\beta}\right)=\operatorname{cl}\left({ }^{\Psi_{\alpha}^{\gamma} \mu_{\alpha}\left(m_{\alpha}\right)} \Phi_{\beta}^{\gamma}\left(m_{\beta}\right)\right) \\
& \left.=\operatorname{cl}\left({ }^{\mu_{\gamma}} \Phi_{\alpha}^{\gamma}\left(m_{\alpha}\right) \Phi_{\beta}^{\gamma}\left(m_{\beta}\right)\right)=\operatorname{cl}\left(\Phi_{\alpha}^{\gamma}\left(m_{\alpha}\right), \Phi_{\beta}^{\gamma}\left(m_{\beta}\right)\right]\right) \\
& =\left[\operatorname{cl}\left(m_{\alpha}\right), \operatorname{cl}\left(m_{\beta}\right)\right], \quad \text { where } \gamma \geqslant \alpha, \beta .
\end{aligned}
$$

Hence $\mu$ is a crossed $\lim _{\alpha}\left\{P_{\alpha}\right\}$-module.

Suppose

$$
\begin{gathered}
t:\left(\lim _{\longrightarrow}\left\{M_{\alpha}\right\}\right) \otimes^{q} N \longrightarrow \underline{\lim }_{\alpha}\left\{M_{\alpha} \otimes^{q} N\right\} \\
\text { (resp. } \left.t:\left(\lim _{\alpha}\left\{M_{\alpha}\right\}\right) \wedge^{q} N \longrightarrow \underline{\lim }_{\alpha}\left\{M_{\alpha} \wedge^{q} N\right\}\right)
\end{gathered}
$$

is a homomorphism defined by the formula $t\left(c l\left(m_{\alpha}\right) \otimes n\right)=\operatorname{cl}\left(m_{\alpha} \otimes n\right)\left(\operatorname{resp} . t\left(\operatorname{cl}\left(m_{\alpha}\right) \wedge n\right)=\right.$ $\left.c l\left(m_{\alpha} \wedge n\right)\right)$ and $t\left(\left\{\left(c l\left(m_{\alpha}\right), n\right)\right\}\right)=\operatorname{cl}\left(\left\{\left(\Phi_{\alpha}^{\beta}\left(m_{\alpha}\right), n\right)\right\}\right)$. Note that there is a $\beta \geqslant \alpha$ such that $\left(\Phi_{\alpha}^{\beta}\left(m_{\alpha}\right), n\right) \in M_{\beta} \times_{P_{\beta}} N$, when $\left(\operatorname{cl}\left(m_{\alpha}\right), n\right) \in\left(\varliminf_{\alpha} M_{\alpha}\right) \times_{\varliminf_{\alpha} P_{\alpha}} N$. To prove that $t$ is well defined we repeat the corresponding part of the proof of Theorem 1.5 in [15].

It is clear that $t$ commutes with relations (1.1) and (1.2). Let us show the compatibility with relations (1.3)-(1.9).

$$
\begin{aligned}
& t\left(\left[\operatorname{cl}\left(m_{\alpha}\right), \operatorname{cl}\left(m_{\beta}\right)\right] \otimes n\right)=t\left(\operatorname{cl}\left(\left[\Phi_{\alpha}^{\gamma}\left(m_{\alpha}\right), \Phi_{\beta}^{\gamma}\left(m_{\beta}\right)\right]\right) \otimes n\right) \\
& =\operatorname{cl}\left(\left[\Phi_{\alpha}^{\gamma}\left(m_{\alpha}\right), \Phi_{\beta}^{\gamma}\left(m_{\beta}\right)\right] \otimes n\right) \\
& =\operatorname{cl}\left(\Phi_{\alpha}^{\gamma}\left(m_{\alpha}\right) \otimes^{\mu_{\gamma} \Phi_{\beta}^{\gamma}\left(m_{\beta}\right)} n-\Phi_{\beta}^{\gamma}\left(m_{\beta}\right) \otimes^{\mu_{\gamma} \Phi_{\alpha}^{\gamma}\left(m_{\alpha}\right)} n\right) \\
& =\operatorname{cl}\left(\left(\Phi_{\alpha}^{\gamma} \otimes^{q} 1_{N}\right)\left(m_{\alpha} \otimes \Psi_{\beta}^{\gamma} \mu_{\beta}\left(m_{\beta}\right) n\right)-\left(\Phi_{\beta}^{\gamma} \otimes^{q} 1_{N}\right)\left(m_{\beta} \otimes \Psi_{\alpha}^{\gamma} \mu_{\alpha}\left(m_{\alpha}\right) n\right)\right) \\
& =c l\left(m_{\alpha} \otimes \mu_{\beta}\left(m_{\beta}\right) n-c l\left(m_{\beta} \otimes \mu_{\alpha}\left(m_{\alpha}\right) n\right)\right. \\
& =t\left(c l\left(m_{\alpha}\right) \otimes^{c l\left(m_{\beta}\right)} n-c l\left(m_{\beta}\right) \otimes{ }^{c l\left(m_{\alpha}\right)} n\right) \text {, for some } \gamma \geqslant \alpha, \beta \text {. }
\end{aligned}
$$

Similarly it can be proved that $t$ preserves the second relation of (1.3). Next

$$
\begin{aligned}
& t\left(\left[\operatorname{cl}\left(m_{\alpha}\right) \otimes n, \operatorname{cl}\left(m_{\beta}\right) \otimes n^{\prime}\right]\right)=\left[\operatorname{cl}\left(m_{\alpha} \otimes n\right), \operatorname{cl}\left(m_{\beta} \otimes n^{\prime}\right)\right] \\
& =\operatorname{cl}\left(\left[\left(\Phi_{\alpha}^{\gamma} \otimes^{q} 1_{N}\right)\left(m_{\alpha} \otimes n\right),\left(\Phi_{\beta}^{\gamma} \otimes^{q} 1_{N}\right)\left(m_{\beta} \otimes n^{\prime}\right)\right]\right) \\
& =c l\left(\left[\Phi_{\alpha}^{\gamma}\left(m_{\alpha}\right) \otimes n, \Phi_{\beta}^{\gamma}\left(m_{\beta}\right) \otimes n^{\prime}\right]\right)=-c l\left({ }^{n} \Phi_{\alpha}^{\gamma}\left(m_{\alpha}\right) \otimes{ }_{\beta}^{\gamma}\left(m_{\beta}\right) n^{\prime}\right) \\
& =-t\left({ }^{n} c l\left(m_{\alpha}\right) \otimes{ }^{c l\left(m_{\beta}\right)} n^{\prime}\right), \quad \gamma \geqslant \alpha, \beta, \\
& t\left(\left[\left\{\left(\operatorname{cl}\left(m_{\alpha}\right), n\right)\right\}, \operatorname{cl}\left(m_{\beta}\right) \otimes n^{\prime}\right]\right)=\left[\operatorname{cl}\left(\left\{\left(\Phi_{\alpha}^{\gamma}\left(m_{\alpha}\right), n\right)\right\}\right), \operatorname{cl}\left(m_{\beta} \otimes n^{\prime}\right)\right] \\
& =\operatorname{cl}\left(\left[\left(\Phi_{\gamma}^{\gamma^{\prime}} \otimes^{q} 1_{N}\right)\left(\left\{\left(\Phi_{\alpha}^{\gamma}\left(m_{\alpha}\right), n\right)\right\}\right), \Phi_{\beta}^{\gamma^{\prime}}\left(m_{\beta} \otimes n^{\prime}\right)\right]\right) \\
& =\operatorname{cl}\left(\left[\left\{\left(\Phi_{\alpha}^{\gamma^{\prime}}\left(m_{\alpha}\right), n\right)\right\}, \Phi_{\beta}^{\gamma^{\prime}}\left(m_{\beta}\right) \otimes n^{\prime}\right]\right) \\
& =c l\left({ }^{q n} \Phi_{\beta}^{\gamma^{\prime}}\left(m_{\beta}\right) \otimes n^{\prime}+\Phi_{\beta}^{\gamma^{\prime}}\left(m_{\beta}\right) \otimes{ }^{q n} n^{\prime}\right) \\
& =t\left({ }^{q n} \operatorname{cl}\left(m_{\beta}\right) \otimes n^{\prime}+c l\left(m_{\beta}\right) \otimes{ }^{q n} n^{\prime}\right), \quad \gamma^{\prime} \geqslant \gamma, \beta, \\
& t\left(\left\{\lambda\left(c l\left(m_{\alpha}\right), n\right)+\lambda^{\prime}\left(c l\left(m_{\beta}\right), n^{\prime}\right)\right\}\right) \\
& =t\left(\left\{\left(\operatorname{cl}\left(\Phi_{\alpha}^{\gamma}\left(\lambda m_{\alpha}\right)+\Phi_{\beta}^{\gamma}\left(\lambda^{\prime} m_{\beta}\right)\right), \lambda n+\lambda^{\prime} n^{\prime}\right)\right\}\right) \\
& =\operatorname{cl}\left(\left\{\left(\Phi_{\gamma}^{\gamma^{\prime}}\left(\Phi_{\alpha}^{\gamma}\left(\lambda m_{\alpha}\right)+\Phi_{\beta}^{\gamma}\left(\lambda^{\prime} m_{\beta}\right)\right), \lambda n+\lambda^{\prime} n^{\prime}\right)\right\}\right) \\
& =\operatorname{cl}\left(\left\{\left(\Phi_{\alpha}^{\gamma^{\prime}}\left(\lambda m_{\alpha}\right)+\Phi_{\beta}^{\gamma^{\prime}}\left(\lambda^{\prime} m_{\beta}\right)\right), \lambda n+\lambda^{\prime} n^{\prime}\right)\right\} \\
& =\operatorname{cl}\left(\lambda\left\{\left(\Phi_{\alpha}^{\gamma^{\prime}}\left(m_{\alpha}\right), n\right)\right\}+\lambda^{\prime}\left(\left\{\Phi_{\beta}^{\gamma^{\prime}}\left(m_{\beta}\right), n^{\prime}\right)\right\}\right) \\
& =t\left(\lambda\left\{\left(\operatorname{cl}\left(m_{\alpha}\right), n\right)\right\}+\lambda^{\prime}\left\{\left(\operatorname{cl}\left(m_{\beta}\right), n^{\prime}\right)\right\}\right),
\end{aligned}
$$




$$
\begin{gathered}
t\left(\left[\left\{\left(c l\left(m_{\alpha}\right), n\right)\right\},\left\{\left(\operatorname{cl}\left(m_{\beta}\right), n^{\prime}\right)\right\}\right]\right) \\
=\left[\operatorname{cl}\left(\left\{\left(\Phi_{\alpha}^{\gamma}\left(m_{\alpha}\right), n\right)\right\}, \operatorname{cl}\left(\left\{\left(\Phi_{\beta}^{\gamma^{\prime}}\left(m_{\beta}\right), n^{\prime}\right)\right\}\right)\right]\right. \\
=\operatorname{cl}\left(\left[\left\{\left(\Phi_{\alpha}^{\gamma^{\prime \prime}}\left(m_{\alpha}\right), n\right)\right\},\left\{\left(\Phi_{\beta}^{\gamma^{\prime \prime}}\left(m_{\beta}\right), n^{\prime}\right)\right\}\right]\right) \\
=\operatorname{cl}\left(q \Phi_{\alpha}^{\gamma^{\prime \prime}}\left(m_{\alpha}\right) \otimes q n^{\prime}\right)=t\left(q \cdot c l\left(m_{\alpha}\right) \otimes q n^{\prime}\right), \\
t\left(\left\{\left(-{ }^{n} \operatorname{cl}\left(m_{\alpha}\right),{ }^{c l}\left(m_{\alpha}\right) n\right)\right\}\right)=t\left(\left\{\left(c l\left(-{ }^{n} m_{\alpha}\right), m_{\alpha} n\right)\right\}\right) \\
=\operatorname{cl}\left(\left\{\left(\Phi_{\alpha}^{\beta}\left(-{ }^{n} m_{\alpha}\right),{ }^{m_{\alpha}} n\right)\right\}\right)=\operatorname{cl}\left(\left\{\left(-{ }^{n} \Phi_{\alpha}^{\beta}\left(m_{\alpha}\right),{ }^{\beta}{ }^{\beta}\left(m_{\alpha}\right) n\right)\right\}\right) \\
=\operatorname{cl}\left(q\left(\Phi_{\alpha}^{\beta}\left(m_{\alpha}\right) \otimes n\right)\right)=t\left(q\left(\operatorname{cl}\left(m_{\alpha}\right) \otimes n\right)\right) .
\end{gathered}
$$

If $c l\left(\mu_{\alpha}\left(m_{\alpha}\right)\right)=\operatorname{cl}\left(\nu_{\alpha}(n)\right)$ then there is $\beta \geqslant \alpha$ such that $\Psi_{\alpha}^{\beta} \mu_{\alpha}\left(m_{\alpha}\right)=\Psi_{\alpha}^{\beta} \nu_{\alpha}(n)$ and hence $\mu_{\beta} \Phi_{\alpha}^{\beta}\left(m_{\alpha}\right)=\nu_{\beta}(n)$. Then

$$
t\left(c l\left(m_{\alpha}\right) \wedge n\right)=\operatorname{cl}\left(m_{\alpha} \wedge n\right)=\operatorname{cl}\left(\Phi_{\alpha}^{\beta}\left(m_{\alpha}\right) \wedge n\right)=0 .
$$

On the other hand, the homomorphisms

$$
\begin{gathered}
\Phi_{\alpha} \otimes^{q} 1_{N}: M_{\alpha} \otimes^{q} N \rightarrow\left(\lim _{\alpha}\left\{M_{\alpha}\right\}\right) \otimes^{q} N \\
\left(\text { resp. } \Phi_{\alpha} \wedge^{q} 1_{N}: M_{\alpha} \wedge^{q} N \rightarrow\left(\lim _{\longrightarrow}\left\{M_{\alpha}\right\}\right) \wedge^{q} N\right),
\end{gathered}
$$

where $\Phi_{\alpha}: M_{\alpha} \rightarrow \lim _{\alpha}\left\{M_{\alpha}\right\}$ are the canonical homomorphisms, induce a homomorphism $t^{\prime}: \lim _{\longrightarrow}\left\{M_{\alpha} \otimes^{q} N\right\} \longrightarrow\left(\lim _{\longrightarrow}\left\{M_{\alpha}\right\}\right) \otimes^{q} N\left(\right.$ resp. $\left.t^{\prime}: \varliminf_{\longrightarrow}\left\{M_{\alpha} \wedge^{q} N\right\} \rightarrow\left(\lim _{\alpha}\left\{M_{\alpha}\right\}\right) \wedge^{q} N\right)$. It is easy to see that $t t^{\prime}, \overrightarrow{t^{\prime} t}$ are identity maps.

One has the following generalization of the homomorphism $\varphi$ in Proposition 1.6

Theorem 1.10. (i) Let $p$ be a positive integer and let $q^{\prime}=p q$. Then there is a Lie homomorphism $\varphi^{\prime}: M \otimes^{q^{\prime}} N \rightarrow M \otimes^{q} N$ given by

$$
\varphi^{\prime}(m \otimes n)=m \otimes n, \quad \varphi^{\prime}(\{k\})=\{p k\}
$$

for $m \in M, n \in N, k \in K$. Furthermore, the Lie homomorphism $\varphi^{\prime}$ induces a Lie homomorphism $\psi^{\prime}: M \wedge^{q^{\prime}} N \rightarrow M \wedge^{q} N$.

(ii) Let $L=K /[M, N]$ then coker $\varphi^{\prime}$ and coker $\psi^{\prime}$ are isomorphic to $L / p L$.

Proof. (i) We have to show that $\varphi^{\prime}$ commutes with relations (1.1)-(1.9). It is clear for relations (1.1)-(1.4) and (1.9). Now, for $m \in M, n \in N, k, k^{\prime} \in K, \lambda, \lambda^{\prime} \in \Lambda$ we have

$$
\begin{gathered}
\varphi^{\prime}([\{k\}, m \otimes n])=[\{p k\}, m \otimes n]={ }^{q p k} m \otimes n+m \otimes{ }^{q p k} n \\
={ }^{q^{\prime} k} m \otimes n+m \otimes q^{\prime} k n=\varphi^{\prime}\left(q^{\prime} k m \otimes n+m \otimes q^{\prime} k\right) ; \\
\varphi^{\prime}\left(\left\{\lambda k+\lambda^{\prime} k^{\prime}\right\}\right)=\left\{p\left(\lambda k+\lambda^{\prime} k^{\prime}\right)\right\}=\lambda\{p k\}+\lambda^{\prime}\left\{p k^{\prime}\right\}=\varphi^{\prime}\left(\lambda\{k\}+\lambda^{\prime}\left\{k^{\prime}\right\}\right) ; \\
\varphi^{\prime}\left(\left[\{k\},\left\{k^{\prime}\right\}\right]\right)=\left[\{p k\},\left\{p k^{\prime}\right\}\right]=\pi_{1}(q p k) \otimes \pi_{2}\left(q p k^{\prime}\right) \\
=\varphi^{\prime}\left(\pi_{1}\left(q^{\prime} k\right) \otimes \pi_{2}\left(q^{\prime} k^{\prime}\right)\right) ; \\
\varphi^{\prime}\left(\left\{\left(-{ }^{n} m,{ }^{m} n\right)\right\}\right)=\left\{p\left(-{ }^{n} m,{ }^{m} n\right)\right\}=q q^{\prime}(m \otimes n)=\varphi^{\prime}\left(q^{\prime}(m \otimes n)\right) .
\end{gathered}
$$

(ii) Can be proved by analogy with the group theoretic version (see [5, Theorem 1.22]).

Suppose that $\mu: M \rightarrow P$ and $\nu: N \rightarrow P$ are two crossed $P$-modules in the context of Lie algebras. There is an action of $P$ on $M \otimes^{q} N\left(M \wedge^{q} N\right)$ which on generators is given by ${ }^{p}(m \otimes n)={ }^{p} m \otimes n+m \otimes{ }^{p} n\left({ }^{p}(m \wedge n)={ }^{p} m \wedge n+m \wedge{ }^{p} n\right)$ and ${ }^{p}\{k\}=\left\{{ }^{p} k\right\}$ for $m \in M$, $n \in N, k \in K$ and $p \in P$. The proof of this fact is left for the reader.

Now we give the definition of the crossed square of Lie algebras which is the crossed 2-cube of Lie algebras (see [8, Definitions 1.3 and 1.4]). 
Definition 1.11. A crossed square is a commutative diagram of Lie algebras

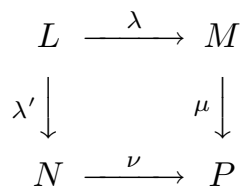

endowed with an action of $P$ on each Lie algebra and a bilinear function $h: M \times N \longrightarrow L$ such that

(i) $\mu, \quad \nu$ and $\alpha=\nu \lambda^{\prime}=\mu \lambda$ are crossed modules, and the maps $\lambda, \lambda^{\prime}$ preserve the actions of $P$;

(ii) $\lambda h(m, n)=-{ }^{n} m, \lambda^{\prime} h(m, n)={ }^{m} n$;

(iii) $h(\lambda(l), n)=-{ }^{n} l, h\left(m, \lambda^{\prime}(l)\right)={ }^{m} l$;

(iv) $h\left(\left[m, m^{\prime}\right], n\right)=h\left(m,{ }^{m^{\prime}} n\right)-h\left(m^{\prime},{ }^{m} n\right)$, $h\left(m,\left[n, n^{\prime}\right]\right)=h\left({ }^{n^{\prime}} m, n\right)-h\left({ }^{n} m, n^{\prime}\right)$;

(v) ${ }^{p} h(m, n)=h\left({ }^{p} m, n\right)+h\left(m,{ }^{p} n\right)$

for all $m, m^{\prime} \in M, n, n^{\prime} \in N, p \in P, l \in L$.

It is easy to obtain the following property of crossed squares of Lie algebras:

Lemma 1.12. Consider a crossed square of Lie algebras. Then:

(i) with the actions induced by the image in $P$ the morphisms $\lambda, \lambda^{\prime}$ are crossed modules;

(ii) the actions of $M$ on $\operatorname{Ker} \lambda^{\prime}$ and of $N$ on $\operatorname{Ker} \lambda$ are trivial;

(iii) $h\left(\lambda(l), \lambda^{\prime}\left(l^{\prime}\right)\right)=\left[l, l^{\prime}\right]$ for all $l, l^{\prime} \in L$.

Now we list some examples of crossed squares of Lie algebras. Throughout, $P$ is an arbitrary Lie algebra and $\mu: M \rightarrow P$ and $\nu: N \rightarrow P$ are crossed $P$-modules.

(1) The square (pull)

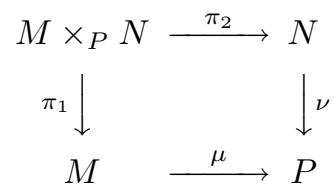

with $h(m, n)=\left(-{ }^{n} m,{ }^{m} n\right)$ is a crossed square.

(2) The square

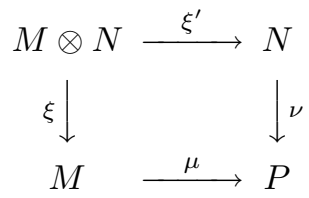

with $\xi(m \otimes n)=-{ }^{n} m, \xi^{\prime}(m \otimes n)={ }^{m} n$ and $h(m, n)=m \otimes n$ is a crossed square.

(3) The square

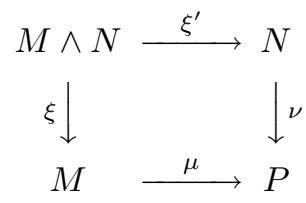

with $h(m, n)=m \wedge n$ is a crossed square.

One has the following 
Lemma 1.13. For $m \in M, n \in N, k \in K$ we have the following relations:

$$
\begin{gathered}
m\{k\}=m \otimes \pi_{2}(q k), \\
{ }^{n}\{k\}=-\pi_{1}(q k) \otimes n .
\end{gathered}
$$

Proof. ${ }^{m}\{k\}=\left\{\left(\left[m, \pi_{1}(k)\right],{ }^{m} \pi_{2}(k)\right)\right\}=\left\{\left(-\pi_{2}(k) m,{ }^{m} \pi_{2}(k)\right)\right\}=m \otimes \pi_{2}(q k)$ by formula (1.8).

The proof of the second formula is similar.

Now we have a fourth example of a crossed square of Lie algebras

Proposition 1.14. The square

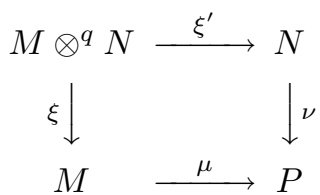

is a crossed square with the function $h$ given by $h(m, n)=m \otimes n$, where $\xi$ and $\xi^{\prime}$ are the Lie homomorphisms defined in Proposition 1.3.

Proof. We have to check each property of a crossed square.

(i) It is easy to see that $\xi$ and $\xi^{\prime}$ preserve the actions of $P$. Consider $\alpha=\mu \xi=\nu \xi^{\prime}$, then:

$$
\begin{gathered}
\alpha\left({ }^{p}(m \otimes n)\right)=\mu \xi\left({ }^{p} m \otimes n+m \otimes{ }^{p} n\right)=\mu\left(-{ }^{n}\left({ }^{p} m\right)-{ }^{\left.{ }^{p} n\right)} m\right) \\
=\mu\left(-{ }^{p}\left({ }^{n} m\right)\right)=\left[p, \mu\left(-{ }^{n} m\right)\right]=[p, \alpha(m \otimes n)], \\
\alpha\left({ }^{p}\{k\}\right)=\mu \xi\left(\left\{{ }^{p} k\right\}\right)=\mu \pi_{1}\left({ }^{p}(q k)\right)=\left[p, \mu \pi_{1}(q k)\right]=[p, \alpha(\{k\})], \\
\alpha(m \otimes n)\left(m^{\prime} \otimes n^{\prime}\right)={ }^{\mu\left(-{ }^{n} m\right)}\left(m^{\prime} \otimes n^{\prime}\right)=[\mu(m), \nu(n)] m^{\prime} \otimes n^{\prime}+m^{\prime} \otimes[\mu(m), \nu(n)] n^{\prime} \\
=-{ }^{n}\left[m, m^{\prime}\right] \otimes n^{\prime}+\left[m,{ }^{n} m^{\prime}\right] \otimes n^{\prime}-m^{\prime} \otimes\left[n,{ }^{m} n^{\prime}\right]+m^{\prime} \otimes{ }^{m}\left[n, n^{\prime}\right] \\
=-\left[{ }^{n} m, m^{\prime}\right] \otimes n^{\prime}+m^{\prime} \otimes\left[{ }^{m} n, n^{\prime}\right] \\
=-{ }^{n} m \otimes m^{\prime} n^{\prime}-m^{\prime} \otimes\left[{ }^{m} n, n^{\prime}\right]+m^{\prime} \otimes\left[{ }^{m} n, n^{\prime}\right]=\left[m \otimes n, m^{\prime} \otimes n^{\prime}\right]
\end{gathered}
$$

by formulas (1.3) and (1.4),

$$
\begin{aligned}
& \alpha(m \otimes n)\{k\}={ }^{m}\left({ }^{n}\{k\}\right)-{ }^{n}\left({ }^{m}\{k\}\right)={ }^{m}\left(-\pi_{1}(q k) \otimes n\right)-{ }^{n}\left(m \otimes \pi_{2}(q k)\right) \\
& =\left[\pi_{1}(q k), m\right] \otimes n-\pi_{1}(q k) \otimes{ }^{m} n-{ }^{n} m \otimes \pi_{2}(q k)-m \otimes\left[n, \pi_{2}(q k)\right] \\
& =\pi_{1}(q k) \otimes{ }^{m} n-m \otimes{ }^{q k} n-\pi_{1}(q k) \otimes{ }^{m} n-{ }^{n} m \otimes \pi_{2}(q k)-{ }^{q k} m \otimes n \\
& +{ }^{n} m \otimes \pi_{2}(q k)=[m \otimes n,\{k\}]
\end{aligned}
$$

by Lemma 1.13 and formulas (1.3) and (1.5),

$$
\alpha(\{k\})(m \otimes n)={ }^{\pi_{1}(q k)} m \otimes n+m \otimes{ }^{\pi_{1}(q k)} n=[\{k\}, m \otimes n]
$$

by formula (1.5),

$$
\alpha(\{k\})\left\{k^{\prime}\right\}={ }^{\pi_{1}(q k)}\left\{k^{\prime}\right\}=\pi_{1}(q k) \otimes \pi_{2}\left(q k^{\prime}\right)=\left[\{k\},\left\{k^{\prime}\right\}\right]
$$

by Lemma 1.13 and formula (1.7).

Now if $x, y \in M \otimes \otimes^{q} N$ are such that $\alpha\left({ }^{p} x\right)=[p, \alpha(x)]$ and $\alpha\left({ }^{p} y\right)=[p, \alpha(y)]$, then

$$
\begin{aligned}
& \alpha\left({ }^{p}[x, y]\right)=\left[\alpha\left({ }^{p} x\right), \alpha(y)\right]+\left[\alpha(x), \alpha\left({ }^{p} y\right)\right] \\
& =[[p, \alpha(x)], \alpha(y)]+[\alpha(x),[p, \alpha(y)]]=[p, \alpha([x, y])] ;
\end{aligned}
$$

Next, if $x_{1}, y_{1}, z_{1} \in M \otimes^{q} N$ are such that ${ }^{\alpha\left(x_{1}\right)} y_{1}=\left[x_{1}, y_{1}\right]$ and ${ }^{\alpha\left(x_{1}\right)} z_{1}=\left[x_{1}, z_{1}\right]$, then

$$
{ }^{\alpha\left(x_{1}\right)}\left[y_{1}, z_{1}\right]=\left[\left[x_{1}, y_{1}\right], z_{1}\right]+\left[y_{1},\left[x_{1}, z_{1}\right]\right]=\left[x_{1},\left[y_{1}, z_{1}\right]\right] ;
$$


And finally, if $x_{2}, y_{2}, z_{2} \in M \otimes^{q} N$ are such that ${ }^{\alpha\left(x_{2}\right)} z_{2}=\left[x_{2}, z_{2}\right]$ and ${ }^{\alpha\left(y_{2}\right)} z_{2}=\left[y_{2}, z_{2}\right]$, then

$$
\begin{aligned}
& \alpha\left(\left[x_{2}, y_{2}\right]\right) z_{2}={ }^{\alpha\left(x_{2}\right)}\left({ }^{\alpha\left(y_{2}\right)} z_{2}\right)-{ }^{\alpha\left(y_{2}\right)}\left({ }^{\alpha\left(x_{2}\right)} z_{2}\right) \\
& =\left[x_{2},\left[y_{2}, z_{2}\right]\right]-\left[y_{2},\left[x_{2}, z_{2}\right]\right]=\left[\left[x_{2}, y_{2}\right], z_{2}\right] .
\end{aligned}
$$

Thus $\alpha$ is a crossed module.

(ii), (iv) and (v) are clear.

(iii) For the proof of the first formula we consider the two cases $l=m \otimes n$ and $l=\{k\}$, then one has

$$
\xi(m \otimes n) \otimes n^{\prime}=-{ }^{n} m \otimes n^{\prime}=-{ }^{n^{\prime}} m \otimes n+m \otimes\left[n, n^{\prime}\right]=-{ }^{n^{\prime}}(m \otimes n)
$$

by formula (1.3),

$$
\xi(\{k\}) \otimes n=\pi_{1}(q k) \otimes n=-{ }^{n}\{k\}
$$

by Lemma 1.13, and observe that if $\xi(l) \otimes n=-{ }^{n} l$ and $\xi\left(l^{\prime}\right) \otimes n=-{ }^{n} l^{\prime}$, for $l, l^{\prime} \in M \otimes \otimes^{q} N$, then by formula (1.3) and (i) it can be written

$$
\begin{aligned}
& \xi\left(\left[l, l^{\prime}\right]\right) \otimes n=\xi(l) \otimes^{\xi\left(l^{\prime}\right)} n-\xi\left(l^{\prime}\right) \otimes^{\xi(l)} n \\
& ={ }^{\xi\left(l^{\prime}\right)}(\xi(l) \otimes n)-{ }^{\xi\left(l^{\prime}\right)} \xi(l) \otimes n-\xi(l)\left(\xi\left(l^{\prime}\right) \otimes n\right)+{ }^{\xi(l)} \xi\left(l^{\prime}\right) \otimes n \\
& =\left[l^{\prime},-{ }^{n} l\right]+\xi\left(\left[l, l^{\prime}\right]\right) \otimes n-\left[l,-{ }^{n} l^{\prime}\right]+\xi\left(\left[l, l^{\prime}\right]\right) \otimes n,
\end{aligned}
$$

so that

$$
\xi\left(\left[l, l^{\prime}\right]\right) \otimes n=-\left(\left[l,{ }^{n} l^{\prime}\right]+\left[{ }^{n} l, l^{\prime}\right]\right)=-{ }^{n}\left[l, l^{\prime}\right] .
$$

The proof of the second formula is similar. $\square$

Corollary 1.15. The square

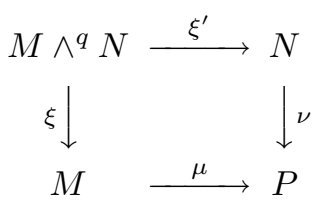

with $h(m, n)=m \wedge n$ is a crossed square.

Lemma 1.16. (i) With the action induced by the image of $K=M \times_{P} N$ in $P$ the Lie homomorphism $\delta: M \otimes^{q} N \rightarrow K\left(\delta^{\prime}: M \wedge^{q} N \rightarrow K\right)$, constructed in Remark 1.4 is a crossed module.

(ii) If $x \in M \wedge^{q} N$ (resp. $x \in M \otimes^{q} N$ ), then $\left\{\delta^{\prime}(x)\right\}=q x$ (resp. $\left.\{\delta(x)\}=q x\right)$.

Proof. (i) immediately follows from the direct calculations.

(ii) For $k \in K$ by formula (1.6)

$$
\left\{\delta^{\prime}(\{k\})\right\}=\left\{\left(\pi_{1}(q k), \pi_{2}(q k)\right)\right\}=\{q k\}=q\{k\},
$$

for $m \in M$ and $n \in N$, using (1.8) one has

$$
\left\{\delta^{\prime}(m \wedge n)\right\}=\left\{\left(-{ }^{n} m,{ }^{m} n\right)\right\}=q(m \wedge n) .
$$

Now let $x, y \in M \wedge^{q} N, \lambda, \lambda^{\prime} \in \Lambda,\left\{\delta^{\prime}(x)\right\}=q x$ and $\left\{\delta^{\prime}(y)\right\}=q y$, then by formula (1.6)

$$
\left\{\delta^{\prime}\left(\lambda x+\lambda^{\prime} y\right)\right\}=q\left(\lambda x+\lambda^{\prime} y\right) .
$$

Next, by relation (1.8), Lemma 1.12(iii) and Proposition 1.14 one has

$$
\left\{\delta^{\prime}([x, y])\right\}=\left\{\left(-{ }^{\pi_{2} \delta^{\prime}(y)} \pi_{1} \delta^{\prime}(x),{ }^{\pi_{1} \delta^{\prime}(x)} \pi_{2} \delta^{\prime}(y)\right)\right\}=q\left(\xi(x) \wedge \xi^{\prime}(y)\right)=q[x, y] .
$$

It is enough to see that $\left\{\delta^{\prime}(x)\right\}=q x$ for any $x \in M \wedge^{q} N$. The equality $\{\delta(x)\}=q x$ for $x \in M \otimes^{q} N$ can be proved similarly. 
Now we analyse the kernel of the canonical homomorphism $M \otimes^{q} N \rightarrow M \wedge^{q} N$. In order to do this we consider the generalized version of Whitehead's universal quadratic functor $\Gamma$ [19], which is defined by Simson and Tye in [18] (see also [6]) for any $\Lambda$-module $A$ as the $\Lambda$-module $\Gamma(A)$ generated by the symbols $\gamma(a)$ with $a \in A$, subject to the relations

$$
\begin{aligned}
\lambda^{2} \gamma(a) & =\gamma(\lambda a), \\
\gamma(a+b+c)+\gamma(a)+\gamma(b)+\gamma(c) & =\gamma(a+b)+\gamma(a+c)+\gamma(b+c), \\
\gamma(\lambda a+b)+\lambda \gamma(a)+\lambda \gamma(b) & =\lambda \gamma(a+b)+\gamma(\lambda a)+\gamma(b)
\end{aligned}
$$

for all $\lambda, \lambda^{\prime} \in \Lambda, a, b, c \in A$.

Let $\mu: M \rightarrow P$ and $\nu: N \rightarrow P$ be two crossed $P$-modules. Suppose the image of $\left(\xi, \xi^{\prime}\right)$ is written $\langle M, N\rangle$. It is easy to check that $\langle M, N\rangle$ is an ideal of $K=M \times_{P} N$ and the quotient is abelian. One has the following

Theorem 1.17. There is a natural exact sequence of Lie algebras

$$
\Gamma(K /<M, N>) \stackrel{\psi}{\rightarrow} M \otimes^{q} N \stackrel{t}{\rightarrow} M \wedge^{q} N \longrightarrow 0
$$

where $\psi(\gamma(c l(m, n)))=m \otimes n$ and $t(m \otimes n)=m \wedge n$.

Proof. It is easily seen from relations (1.1), (1.4)-(1.7) that any element $x \in M \otimes^{q} N$ is of the form $x=\sum_{i} m_{i} \otimes n_{i}+\{k\}$, so

$$
\begin{aligned}
& \xi(x) \otimes \xi^{\prime}(x)=\xi\left(\sum_{i} m_{i} \otimes n_{i}\right) \otimes \xi^{\prime}\left(\sum_{i} m_{i} \otimes n_{i}\right) \\
& +\xi\left(\sum_{i} m_{i} \otimes n_{i}\right) \otimes \xi^{\prime}(\{k\})+\xi(\{k\}) \otimes \xi^{\prime}\left(\sum_{i} m_{i} \otimes n_{i}\right) \\
& +\xi(\{k\}) \otimes \xi^{\prime}(\{k\}) .
\end{aligned}
$$

But from the proof of Proposition 14 in [6] we have

$$
\xi\left(\sum_{i} m_{i} \otimes n_{i}\right) \otimes \xi^{\prime}\left(\sum_{i} m_{i} \otimes n_{i}\right)=0 .
$$

Next, using Lemma 1.13

$$
\begin{aligned}
& \xi\left(\sum_{i} m_{i} \otimes n_{i}\right) \otimes \xi^{\prime}(\{k\})+\xi(\{k\}) \otimes \xi^{\prime}\left(\sum_{i} m_{i} \otimes n_{i}\right) \\
& =\sum_{i}\left(-{ }^{n_{i}} m_{i} \otimes \pi_{2}(q k)+\pi_{1}(q k) \otimes{ }^{m_{i}} n_{i}\right)=\sum_{i}\left(-{ }^{\left({ }^{n_{i}} m_{i}\right)}\{k\}-{ }^{\left(m_{i} n_{i}\right)}\{k\}\right) \\
& =\sum_{i}\left(-{ }^{\left[\nu\left(n_{i}\right), \mu\left(m_{i}\right)\right]}\{k\}-\left[\mu\left(m_{i}\right), \nu\left(n_{i}\right)\right]\{k\}\right)=0,
\end{aligned}
$$

By relation (1.7)

$$
\xi(\{k\}) \otimes \xi^{\prime}(\{k\})=\pi_{1}(q k) \otimes \pi_{2}(q k)=0 .
$$

So $\xi(x) \otimes \xi^{\prime}(x)=0$ for every $x \in M \otimes^{q} N$. Thus if $c l(m, n)=0$ then $\psi(\gamma(c l(m, n)))=m \otimes n=0$. Clearly $\psi$ commutes with the defining relations of $\Gamma(-)$ and $\operatorname{Im} \psi=M \square N=\operatorname{Ker} t$ (see Definition 1.2). But $M \square N$ is in the centre of $M \otimes^{q} N$ and so $\psi$ is a Lie homomorphism.

Recall the definition of compatible actions of Lie algebras from [6]

Definition 1.18. Let $M$ and $N$ be two Lie algebras with actions on each other. The actions are compatible if

$$
{ }^{\left({ }^{n} m\right)} n^{\prime}=\left[n^{\prime},{ }^{m} n\right] \text { and }{ }^{\left({ }^{m} n\right)} m^{\prime}=\left[m^{\prime},{ }^{n} m\right]
$$

for all $m, m^{\prime} \in M n, n^{\prime} \in N$. 
Let $M$ and $N$ be two Lie algebras with compatible actions on each other. We shall denote by ${ }^{M} N$ the submodule of $N$ generated by the elements of the form ${ }^{m} n, m \in M, n \in N$. It follows from the compatibility condition that ${ }^{M} N$ is an ideal of $N$.

According to the definition of the Peiffer product of groups (see [19],[10]) we have the following

Definition 1.19. The Peiffer product, $M \bowtie N$, of two Lie algebras $M$ and $N$ with compatible actions on each other is the quotient of the coproduct $M * N$ by the relations:

$$
[m, n]={ }^{m} n,[n, m]={ }^{n} m
$$

for all $m \in M, n \in N$.

As a consequence of the compatibility condition the actions of $M * N$ on $M$ and on $N$ factor through $M \bowtie N$ and the canonical maps $M \rightarrow M \bowtie N$ and $N \rightarrow M \bowtie N$ are crossed modules. So we can define an 'absolute' tensor product modulo $q$ of two Lie algebras $M$ and $N$ acting on each other compatibly, by considering them as crossed $M \bowtie N$-modules.

Theorem 1.20. If $M$ and $N$ act trivially on each other (i.e. ${ }^{M} N=\{0\}$ and ${ }^{N} M=\{0\}$ ) then there is an isomorphism

$$
M \otimes^{q} N \approx\left(M^{a b} / q M^{a b}\right) \otimes_{\Lambda / q \Lambda}\left(N^{a b} / q N^{a b}\right),
$$

where $M^{a b}=M /[M, M], N^{a b}=N /[N, N]$ and $[M, M],[N, N]$ are commutants of $M$ and $N$ respectively.

Proof. In the case of trivial actions $[m, n]=0$ in $M \bowtie N$ for all $m \in M, n \in N$ and hence the Peiffer product $M \bowtie N=M \times N$. Clearly $K=M \times_{M \bowtie N} N=0$. So the Lie homomorphism $\varphi$ in Proposition 1.6 is surjective. By [6] in the case of trivial actions one has $M \otimes N \approx M^{a b} \otimes_{\Lambda} N^{a b}$. By relation (1.8) every element in $M \otimes^{q} N$ has an order dividing $q$. Then

$$
\begin{aligned}
& M \otimes^{q} N \approx M \otimes N / q(M \otimes N) \approx M^{a b} \otimes_{\Lambda} N^{a b} / q\left(M^{a b} \otimes_{\Lambda} N^{a b}\right) \\
& \approx\left(M^{a b} / q M^{a b}\right) \otimes_{\Lambda / q \Lambda}\left(N^{a b} / q N^{a b}\right) . \square
\end{aligned}
$$

Now the relation between Ellis' non-abelian tensor product of Lie algebras and the nonabelian tensor product modulo $q$ of Lie algebras with compatible actions on each other will be given, which is the Lie algebra analogue of [15, Theorem 1.9]

First we study the Peiffer product of Lie algebras. Let $M$ and $N$ be two Lie algebras acting compatible on each other and let $\psi: M * N \rightarrow M \bowtie N$ be the natural Lie homomorphism. Then modulo $\operatorname{Ker} \psi,[m, n] \equiv{ }^{m} n$, so that every element of $M \bowtie N$ can be written as $\psi(m)+\psi(n)$ for suitable $m$ and $n$. We denote $\psi(m)+\psi(n)$ by $\langle m, n>$. It is easy to see that the relations

$$
\begin{aligned}
& {\left[<m, n>,<m^{\prime}, n^{\prime}>\right]=<\left[m, m^{\prime}\right]+{ }^{n} m^{\prime}-{ }^{n^{\prime}} m,\left[n, n^{\prime}\right]>} \\
& =<\left[m, m^{\prime}\right],\left[n, n^{\prime}\right]+{ }^{m} n^{\prime}-{ }^{m^{\prime}} n>
\end{aligned}
$$

are defining relations for $M \bowtie N$ on the generators $<m, n>$ and the Peiffer product is a homomorphic image of the semidirect products $M \ltimes N$ and $M \rtimes N$. Furthermore, $M \bowtie N$ is obtaind from $M \ltimes N$ (resp. $M \rtimes N$ ) by imposing the relation

$$
\left({ }^{n} m,{ }^{m} n\right)=0
$$

for all $m \in M$ and $n \in N$, since if $L$ is an ideal of $M \ltimes N$ (resp. $M \rtimes N$ ) generated by the set $\left\{\left({ }^{n} m,{ }^{m} n\right) \mid m \in M, n \in N\right\}$, then we have a Lie homomorphism $M \ltimes N / L \stackrel{\epsilon}{\rightarrow} M \bowtie N$ (resp. $M \rtimes N / L \stackrel{\epsilon}{\rightarrow} M \bowtie N), \epsilon(\operatorname{cl}(m, n))=<m, n>$. On the other hand, there is a Lie homomorphism $M \bowtie N \stackrel{\epsilon^{\prime}}{\rightarrow} M \ltimes N / L$ (resp. $M \bowtie N \stackrel{\epsilon^{\prime}}{\rightarrow} M \rtimes N / L$ ) induced by the canonical homomorphisms $M \rightarrow M \ltimes N$ and $N \rightarrow M \ltimes N$ (resp. $M \rightarrow M \rtimes N$ and $N \rightarrow M \rtimes N$ ). It is clear that $\epsilon \epsilon^{\prime}$ and $\epsilon^{\prime} \epsilon$ are identity maps. 
Let $\mu: M \rightarrow P$ and $\nu: N \rightarrow P$ be two crossed $P$-modules. The actions of $M$ and $N$ on each other via $P$ are always compatible and it is easy to prove the following

Proposition 1.21. There is an exact sequence of Lie algebras

$$
0 \longrightarrow K /[M, N] \stackrel{j}{\rightarrow} M \bowtie N \stackrel{t}{\rightarrow} P
$$

where the map $j$ is induced by the map $K \rightarrow M \ltimes N$ given by $(m, n) \mapsto(m,-n)$ and $t(<m, n>)=\mu(m)+\nu(n)$.

Observe that this result is the Lie algebra analogue of Proposition 2.5 [1] (see also [10], $[15])$.

By (1.10) and Proposition 1.6 one has the following exact sequence of Lie algebras

$$
M \otimes N \stackrel{\varphi}{\rightarrow} M \otimes^{q} N \longrightarrow M \bowtie N \longrightarrow P .
$$

In the case of the 'absolute' tensor product modulo $q$ of Lie algebras $M$ and $N$ acting compatibly on each other and considered as crossed $M \bowtie N$-modules, the natural homomorphism $M \bowtie N \rightarrow P=M \bowtie N$ is the identity map. Thus from (1.11) $\varphi: M \otimes N \rightarrow M \otimes^{q} N$ is an epimorphism and $K=[M, N]$.

Theorem 1.22. Let $M$ and $N$ be Lie algebras equipped with compatible actions on each other. Then there is a short exact sequence of Lie algebras

$$
0 \longrightarrow q\left(\operatorname{Ker} \lambda \cap \operatorname{Ker} \lambda^{\prime}\right) \longrightarrow M \otimes N \stackrel{\varphi}{\rightarrow} M \otimes^{q} N \longrightarrow 0
$$

where $\lambda: M \otimes N \rightarrow M, \lambda^{\prime}: M \otimes N \rightarrow N$ are Lie homomorphisms defined on generators by $\lambda(m \otimes n)=-{ }^{n} m, \lambda^{\prime}(m \otimes n)={ }^{m} n$ (see [6, Proposition 2]).

Proof. Any element $x \in M \otimes N$ is of the form $x=\sum_{i} m_{i} \otimes n_{i}$. Let $x \in \operatorname{Ker} \lambda \cap \operatorname{Ker} \lambda^{\prime}$, then by the formulas (1.8), (1.6) one has

$$
\begin{aligned}
& \varphi(q x)=\sum_{i} q\left(m_{i} \otimes n_{i}\right)=\sum_{i}\left\{\left(-{ }^{n_{i}} m_{i},{ }^{m_{i}} n_{i}\right)\right\} \\
& =\left\{\left(\sum_{i}\left(-{ }^{n_{i}} m_{i}\right), \sum_{i} m_{i} n_{i}\right)\right\}=\left\{\left(\lambda(x), \lambda^{\prime}(x)\right)\right\}=\{(0,0)\}=0 .
\end{aligned}
$$

This proves that $\varphi\left(q\left(\operatorname{Ker} \lambda \cap \operatorname{Ker} \lambda^{\prime}\right)\right)=0$. Hence $\varphi$ induces a natural Lie homomorphism

$$
\psi: M \otimes N / q\left(\operatorname{Ker} \lambda \cap \operatorname{Ker} \lambda^{\prime}\right) \longrightarrow M \otimes^{q} N .
$$

Since $K=[M, N]$ (see above), any element $k \in K$ is of the form $k=\sum_{i}\left(-{ }^{n_{i}} m_{i},{ }^{m_{i}} n_{i}\right)$ for suitable $m_{i} \in M, n_{i} \in N$. Let us define a homomorphism $\psi^{\prime}: M \otimes^{q} N \longrightarrow$ $M \otimes N / q\left(\operatorname{Ker} \lambda \cap \operatorname{Ker} \lambda^{\prime}\right)$ as follows: $\psi^{\prime}(m \otimes n)=\operatorname{cl}(m \otimes n), \psi^{\prime}(\{k\})=\operatorname{cl}\left(\sum_{i} q\left(m_{i} \otimes n_{i}\right)\right)$. It is easy to see that $\psi^{\prime}$ is correctly defined, it preserves the relations (1.1)-(1.8) and $\psi \psi^{\prime}, \psi^{\prime} \psi$ are identity maps.

Note that by [12] if $N \rightarrow M$ (resp. $M \rightarrow N)$ is a crossed $M$-module (resp. $N$-module) then $\operatorname{Ker} \lambda^{\prime}(\operatorname{resp} . \operatorname{Ker} \lambda)$ is the first non-abelian homology $H_{1}(M, N)\left(\operatorname{resp} . H_{1}(N, M)\right)$ of the Lie algebra $M$ (resp. $N)$ with coefficients in the Lie algebra $N$ (resp. $M$ )

Corollary 1.23. If $M$ is a perfect Lie algebra (i.e. $M=[M, M]$ ) then one has the following short exact sequence of Lie algebras

$$
0 \rightarrow q H_{2}(M) \rightarrow M \otimes M \rightarrow M \otimes^{q} M \rightarrow 0
$$

Proof. Follows from Theorem 1.22 and the fact that if $M$ is a perfect Lie algebra then $\operatorname{Ker} \lambda=H_{2}(M)[6$, Theorem 11]. 


\section{The universal $q$-central relative extension of Lie algebras}

Let $M$ and $N$ be two ideals of the Lie algebra $P$, so that there is a canonical identification $K=M \times{ }_{P} N=M \cap N$, sending $(k, k)$ onto $k$. We denote by $M \#_{q} N$ the image of $M \otimes^{q} N$ and $M \wedge^{q} N$ in $K=M \cap N$. Whence $M \#_{q} N$ is the ideal of $K$ generated by elements $[m, n]$ and $q k$ for $m \in M, n \in N$ and $k \in K$.

Proposition 2.1. Suppose that $M$ and $N$ are two ideals of a Lie algebra $P$ and $M \cap N=$ $M \#_{q} N$, then we have

$$
M \otimes^{q} N=M \wedge^{q} N .
$$

Proof. For $k \in K=M \cap N=M \#_{q} N$ there exists $x \in M \otimes^{q} N$ such that $k=\xi(x)$, then by Lemme 1.12(iii) we have

$$
k \otimes k=\xi(x) \otimes \xi(x)=[x, x]=0 .
$$

Now we give the following definition from $[16]$

Definition 2.2. (i) Let $\alpha: P \rightarrow Q$ be a Lie epimorphism and $A$ be a $Q$-module. A relative extension of $\alpha$ by $A$ is an exact sequence of Lie algebras

$$
0 \rightarrow A \rightarrow E \stackrel{\mu}{\rightarrow} P \stackrel{\alpha}{\rightarrow} Q \rightarrow 0
$$

such that $\mu$ is a crossed $P$-module.

(ii) a morphism between the relative extensions

$$
0 \rightarrow A \rightarrow E \stackrel{\mu}{\rightarrow} P \stackrel{\alpha}{\rightarrow} Q \rightarrow 0
$$

and

$$
0 \rightarrow A^{\prime} \rightarrow E^{\prime} \stackrel{\mu^{\prime}}{\rightarrow} P \stackrel{\alpha}{\rightarrow} Q \rightarrow 0
$$

is a P-equivariant Lie homomorphism $\varphi: E \rightarrow E^{\prime}$ (i.e. $\varphi\left({ }^{p} x\right)={ }^{p} \varphi(x)$ for $x \in E, p \in P$ ) such that $\mu^{\prime} \varphi=\mu$.

(iii) A relative extension of $\alpha$ by $A$ is called a central relative extension if $Q$ acts trivially on A.

Following definitions are the Lie algebra analogues of Definitions 2.3-2.5 in [5].

Definition 2.3. A relative extension of $\alpha$ by $A$ is called a $q$-central relative extension if $Q$ acts trivially on $A$ and $q a=0$ for any $a \in A$. Such $q$-central relative extension is called universal if there exists a unique morphism of relative extensions from it to any q-central relative extension of $\alpha$.

Note that if $Q=\{0\}$, then the $q$-central relative extension of $\alpha$ by $A$ is a $q$-central extension of $P$ by $A$, i.e. a central extension of Lie algebras

$$
0 \rightarrow A \rightarrow E \rightarrow P \rightarrow 0
$$

such that $q a=0$ for all $a \in A$.

Definition 2.4. A P-Lie algebra $A$ ( $P$ acts on $A$ ) is called $P$-q-perfect if $A$ is generated by elements of the form $\left[a, a^{\prime}\right]-{ }^{p} a^{\prime}$ and $q a, a, a^{\prime} \in A, p \in P$.

Note that if $P$ acts trivially on $A$, then the $P$-q-perfect Lie algebra $A$ is a $q$-perfect Lie algebra, i.e. $A$ is generated by elements of the form $q a$ and $\left[a^{\prime}, a^{\prime \prime}\right], a, a^{\prime}, a^{\prime \prime} \in A$.

Now we obtain the conditions for the existence of a universal $q$-central relative extension of a Lie epimorphism and describe this extension using exterior (tensor) product modulo $q$. 
Lemma 2.5. Let $P$ be a Lie algebra and $N$ be an ideal of $P$. Then the $P$-Lie algebra $N \wedge^{q} P$ $\left(N \otimes^{q} P\right)$ is $P$-q-perfect if and only if $N=N \#_{q} P$.

Proof. First suppose $N=N \#_{q} P$, then for any $n \in N$ there exists $x \in N \wedge^{q} P$ such that $n=\xi(x)$, thus, by Lemma 1.16(ii) $\{n\}=\{\xi(x)\}=q x$ and by Definition 1.11(iii) and Corollary 1.15 we have $n \wedge p=\xi(x) \wedge p=-{ }^{p} x$. As $N \wedge^{q} P$ is generated by elements $n \wedge p$ and $\{n\}$ it is $P$ - $q$-perfect.

Conversely, let $N \wedge^{q} P$ be $P$-q-perfect. Consider the surjective Lie homomorphism $\psi$ : $N \wedge^{q} P \rightarrow N /[N, P]$ given by Proposition 1.6. As the elements $\left[x, x^{\prime}\right]-{ }^{p} x^{\prime}$ and $q x$ generate $N \wedge^{q} P$, then their images $\psi\left(\left[x, x^{\prime}\right]-{ }^{p} x^{\prime}\right)=\operatorname{cl}\left(\left[\xi\left(x^{\prime}\right), p-\xi(x)\right]\right)=0$ and $\psi(q x)=c l(\xi(q x))$ generate $N /[N, P]$, so $N=N \#_{q} P$.

Lemma 2.6. A short exact sequence of Lie algebras

$$
0 \rightarrow N \rightarrow P \stackrel{\alpha}{\rightarrow} Q \rightarrow 0
$$

gives rise to an exact sequence of Lie algebras

$$
N \wedge^{q} P \stackrel{\xi}{\rightarrow} P \stackrel{\alpha}{\rightarrow} Q \rightarrow 0
$$

if and only if $N=N \#_{q} P$.

Proposition 2.7. Suppose that

$$
0 \rightarrow N \rightarrow P \stackrel{\alpha}{\rightarrow} Q \rightarrow 0
$$

is a short exact sequence of Lie algebras and let

$$
0 \rightarrow A \rightarrow E \stackrel{\mu}{\rightarrow} P \stackrel{\alpha}{\rightarrow} Q \rightarrow 0
$$

be a q-central relative extension of $\alpha$. If $N \neq N \#_{q} P$ then the Lie algebra $E$ is not $P$-q-perfect and this q-central relative extension is not universal.

Proof. If the Lie algebra $E$ is $P$-q-perfect then by surjectivity of the Lie homomorphism $E \stackrel{\mu}{\rightarrow} N$ we obtain that $N$ is $P$-q-perfect i.e. $N=N \#_{q} P$. So $E$ is not $P$-q-perfect.

Let $E_{P}^{q}$ be the submodule of $E$ generated by the elements $\left[x, x^{\prime}\right]-{ }^{p} x^{\prime}$ and $q x, x, x^{\prime} \in E$, $p \in P$. It is easy to see that $E_{P}^{q}$ is an ideal of $E, E / E_{P}^{q} \neq 0$ is abelian and $Q$ acts trivially on $E / E_{P}^{q}$. Then the exact sequence

$$
0 \rightarrow E / E_{P}^{q} \stackrel{i}{\rightarrow} E / E_{P}^{q} \times N \stackrel{\pi}{\rightarrow} P \stackrel{\alpha}{\rightarrow} Q \rightarrow 0
$$

where $\pi(x, n)=n$, is a $q$-central relative extension of $\alpha$.

Let us define Lie homomorphisms $f_{1}, f_{2}: E \rightarrow E / E_{P}^{q} \times N$ as follows: $f_{1}(x)=(\operatorname{cl}(x), \mu(x))$ and $f_{2}(x)=(0, \mu(x))$ for all $x \in E$. Clearly $f_{1}$ and $f_{2}$ are morphisms of relative extensions and $f_{1} \neq f_{2}$. Hence the $q$-central relative extension $0 \rightarrow A \rightarrow E \stackrel{\mu}{\rightarrow} P \stackrel{\alpha}{\rightarrow} Q \rightarrow 0$ is not universal.

Theorem 2.8. Let

$$
0 \rightarrow N \rightarrow P \stackrel{\alpha}{\rightarrow} Q \rightarrow 0
$$

be a short exact sequence of Lie algebras and $N=N \#_{q} P$. Then the exact sequence

$$
0 \rightarrow V \rightarrow N \wedge^{q} P \stackrel{\xi}{\rightarrow} P \stackrel{\alpha}{\rightarrow} Q \rightarrow 0
$$

is the universal $q$-central relative extension of $\alpha$, where $V=K$ Ker $\xi$.

Proof. By Lemme 2.6 this sequence is exact. By Lemma 1.12(ii) and Corollary 1.15 the Lie algebra $Q$ acts trivially on $\operatorname{Ker} \xi$ and by Lemma 1.16(ii) one has $q x=\{\xi(x)\}=0$ for $x \in \operatorname{Ker} \xi$. So the sequence is a $q$-central relative extension of $\alpha$.

Let

$$
0 \rightarrow A \rightarrow E \stackrel{\mu}{\rightarrow} P \stackrel{\alpha}{\rightarrow} Q \rightarrow 0
$$


be another $q$-central relative extension of $\alpha$. Suppose $\vartheta: N \rightarrow E$ be a set-theoretic section of $\mu$ and let us define a map $k: N \wedge^{q} P \rightarrow E$ as follows: $k(n \wedge p)={ }^{p} \vartheta(n)$ and $k(\{n\})=q \vartheta(n)$. We must show that $k$ commutes with relations (1.1) to (1.9).

Clearly

$$
k(n \wedge \lambda p)=-{ }^{\lambda p} \vartheta(n)=\lambda k(n \wedge p) .
$$

Note that if $x, y \in E$ and $x-y \in A$, we have ${ }^{p} x={ }^{p} y$ for all $p \in P$. Then

$$
\begin{gathered}
k(\lambda n \wedge p)=-{ }^{p} \vartheta(\lambda n)=--^{p}(\lambda \vartheta(n))=\lambda k(n \wedge p) ; \\
k\left(\left(n+n^{\prime}\right) \wedge p\right)=-{ }^{p}\left(\vartheta\left(n+n^{\prime}\right)\right)=-{ }^{p}\left(\vartheta(n)+\vartheta\left(n^{\prime}\right)\right)=k(n \wedge p)+k\left(n^{\prime} \wedge p\right) .
\end{gathered}
$$

Clearly

$$
k\left(n \wedge\left(p+p^{\prime}\right)\right)=k(n \wedge p)+k\left(n \wedge p^{\prime}\right)
$$

Using the defining conditions of crossed module

$$
\begin{aligned}
& k\left(\left[n, n^{\prime}\right] \wedge p\right)=-{ }^{p} \vartheta\left(\left[n, n^{\prime}\right]\right)=-{ }^{p}\left[\vartheta(n), \vartheta\left(n^{\prime}\right)\right] \\
& \left.\left.=-\left[{ }^{p} \vartheta(n), \vartheta\left(n^{\prime}\right)\right]-\left[\vartheta(n),{ }^{p} \vartheta\left(n^{\prime}\right)\right]=-{ }^{(p} \vartheta(n)\right) \vartheta\left(n^{\prime}\right)+{ }^{p} \vartheta\left(n^{\prime}\right)\right) \vartheta(n) \\
& =-{ }^{[p, n]} \vartheta\left(n^{\prime}\right)+{ }^{\left[p, n^{\prime}\right]} \vartheta(n)=-k\left(n^{\prime} \wedge{ }^{n} p\right)+k\left(n \wedge^{n^{\prime}} p\right) \text {; }
\end{aligned}
$$

and

$$
\begin{aligned}
& k\left(n \wedge\left[p, p^{\prime}\right]\right)=-\left[{ }^{\left[p, p^{\prime}\right]} \vartheta(n)=-{ }^{p}\left({ }^{p^{\prime}} \vartheta(n)\right)+{ }^{p^{\prime}}\left({ }^{p} \vartheta(n)\right)\right. \\
& =-{ }^{p} \vartheta\left({ }^{p^{\prime}} n\right)+{ }^{p^{\prime}} \vartheta\left({ }^{p} n\right)=k\left({ }^{p^{\prime}} n \wedge p\right)-k\left({ }^{p} n \wedge p^{\prime}\right) .
\end{aligned}
$$

The proof of the commutativity of $k$ with relations (1.4) and (1.5) is similar. Next, since $q x=q y$ for all $x, y \in E$ such that $x-y \in A$, we have

$$
\begin{aligned}
& k\left(\left\{\lambda n+\lambda^{\prime} n^{\prime}\right\}\right)=q \vartheta\left(\lambda n+\lambda^{\prime} n^{\prime}\right)=q\left(\lambda \vartheta(n)+\lambda^{\prime} \vartheta\left(n^{\prime}\right)\right) \\
& =\lambda k(\{n\})+\lambda^{\prime} k\left(\left\{n^{\prime}\right\}\right) ; \\
& \quad k\left(\left[\{n\},\left\{n^{\prime}\right\}\right]\right)=\left[q \vartheta(n), q \vartheta\left(n^{\prime}\right)\right]=-q \vartheta\left(n^{\prime}\right)(q \vartheta(n)) \\
& \quad=-{ }^{q n^{\prime}} \vartheta(q n)=k\left(q n \wedge q n^{\prime}\right) ; \\
& \quad k(\{[n, p]\})=q \vartheta([n, p])=-q^{p} \vartheta(n)=k(q(n \wedge p)) .
\end{aligned}
$$

Finally

$$
k(n \wedge n)=-{ }^{n} \vartheta(n)=-{ }^{\mu \vartheta(n)} \vartheta(n)=-[\vartheta(n), \vartheta(n)]=0 .
$$

Since $\vartheta\left({ }^{p} n\right)-{ }^{p} \vartheta(n) \in A$ one has

$$
k\left({ }^{p}\{n\}\right)=q \vartheta\left({ }^{p} n\right)=q^{p} \vartheta(n)={ }^{p} k(\{n\}) ;
$$

and

$$
k\left({ }^{p}\left(n \wedge p^{\prime}\right)\right)=--^{p^{\prime}} \vartheta\left({ }^{p} n\right)-{ }^{\left[p, p^{\prime}\right]} \vartheta(n)=-{ }^{p}\left(p^{\prime} \vartheta(n)\right)={ }^{p} k\left(n \wedge p^{\prime}\right) .
$$

Thus $k$ is $P$-equivariant.

Suppose $k^{\prime}: N \wedge^{q} P \rightarrow E$ is an other homomorphism such that $\mu k=\mu k^{\prime}=\xi$, then $k(y)-k^{\prime}(y) \in \operatorname{Ker} \mu=A$ and $k(q y)=k^{\prime}(q y)$ for all $y \in N \wedge^{q} P$. On the other hand for any $x, x^{\prime} \in N \wedge^{q} P$ and $p \in P$ we have ${ }^{(\mu(x)-p)}\left(k\left(x^{\prime}\right)-k^{\prime}\left(x^{\prime}\right)\right)=0$ from which comes

$$
k\left(\left[x, x^{\prime}\right]-{ }^{p} x^{\prime}\right)=k^{\prime}\left(\left[x, x^{\prime}\right]-{ }^{p} x^{\prime}\right) .
$$

Thus $k=k^{\prime}$ since $N \wedge^{q} P$ is $P$-q-perfect and is generated by elements $\left[x, x^{\prime}\right]-{ }^{p} x^{\prime}$ and $q y$, for all $x, x^{\prime}, y \in N \wedge^{q} P, p \in P$. 
Remark 2.9. In Theorem 2.8 $N \wedge^{q} P$ can be replaced by $N \otimes^{q} P$.

Corollary 2.10. If $P$ is a q-perfect Lie algebra, then the exact sequence of Lie algebras

$$
0 \rightarrow V \rightarrow P \wedge^{q} P \stackrel{\xi}{\rightarrow} P \rightarrow 0
$$

is the universal q-central extension, where $V=K e r \xi$.

\section{References}

1. R.Brown, Coproducts of crossed P-modules: applications to second homotopy groups and to the homology of groups, Topology 23 (1984), 337-345.

2. R.Brown, q-perfect groups and q-central extensions, Publicaciones Mat. 34 (1990), 291297.

3. R.Brown and J.-L.Loday, Excision homotopique en basse dimension, C.R. Acad. Sci. Paris S.I Math. 298, No 15 (1984), 353-356.

4. R.Brown and J.-L.Loday, Van Kampen theorems for diagrams of spaces, Topology 26 (1987), 311-335.

5. D.Conduche and C.Rodriguez-Fernandez, Non-abelian tensor and exterior products modulo $q$ and universal q-central relative extension, J. Pure Applied Algebra, 78 (1992), 139-160.

6. G.J.Ellis, A non-abelian tensor product of Lie algebras, Glasgow Math. J 33 (1991), 101-120.

7. G.J.Ellis, Non-abelian exterior product of Lie algebras and an exact sequence in the homology of Lie algebras, J. Pure Appl. Algebra 46 (1987), 111-115.

8. G.J.Ellis, Higher dimensional crossed modules of algebras, J. Pure Appl. Algebra bf 52 (1988), 277-282.

9. G.J.Ellis and C.Rodriguez, An exterior product for the homology of groups with integral coefficients modulo p, Cahiers Topologie Géom. Différentielle Catégoriques XXX (1989), 339-344.

10. N.D.Gilbert and P.J.Higgins, The non-abelian tensor product of groups and related constructions, Glasgow Math. J 31 (1989), 17-29.

11. D.Guin, Cohomologie et homologie non-abeliennes des groupes, J. Pure Appl. Algebra 50 (1988), 109-137.

12. D.Guin, Cohomologie des algebres de Lie croisees et K-theorie de Milnor additive, Ann. Inst. Fourier, Grenoble 45 (1995), 93-118.

13. H.Inassaridze and N.Inassaridze, Non-abelian homology of groups, K-Theory J. 378 (1998), 1-17.

14. N.Inassaridze, Non-abelian tensor products and non-abelian homology of groups, J.Pure Appl. Algebra, 112 (1996), 191-205.

15. N.Inassaridze, q-Homology of groups, J.Pure Appl. Algebra (1999) (to appear).

16. C.Kassel and J.-L.Loday, Extensions centrales d'algebres de Lie, Ann. Inst. Fourier (Grenoble) 33 (1982), 119-142.

17. J.-L.Loday, Cohomologie et groupe de Steinberg relatif, J. Algebra 54 (1978), 178-202.

18. D.Simson and A.Tye, Connected sequences of stable derived functors and their applications, Dissertationes Mathematicae (Rozprawy Mat.) 111 (1974).

19. J.H.C.Whitehead, On adding relations to homotopy groups, Ann. of Math. 42 (1941), 409-428. 
This article may be accessed via WWW at http://www.rmi.acnet.ge/hha/ or by anonymous ftp at ftp://ftp.rmi.acnet.ge/pub/hha/volumes/1999/n9/n9.(dvi,ps,dvi.gz,ps.gz)

Emzar Khmaladze khmal@rmi.acnet.ge

A. Razmadze Mathematical Institute Georgian Academy of Sciences

1, M. Aleksidze St., Tbilisi 380093

Georgia 